\title{
Lasting immune memory against hepatitis $B$ in children after primary immunization with 4 doses of DTPa-HBV-IPV/Hib in the first and $2^{\text {nd }}$ year of life
}

Michael Steiner ${ }^{1}$, Gunasekaran Ramakrishnan ${ }^{2}$, Britta Gartner ${ }^{3}$, Olivier Van Der Meeren ${ }^{4}$, Jeanne-Marie Jacquet ${ }^{4}$, Volker Schuster ${ }^{5^{*}}$

\begin{abstract}
Background: Few studies have assessed long term persisting immunity against hepatitis B virus (HBV) in children vaccinated during infancy with combined vaccines containing recombinant HBV surface antigen (HBs). We assessed antibody persistence and immune memory in children 4-5 years of age, previously vaccinated with four doses of combined hexavalent DTPa-HBV-IPV/Hib vaccine (Infanrix hexa ${ }^{\text {TM }}$ ).
\end{abstract}

Methods: Immune memory was assessed in 301 children through administration of a challenge dose of monovalent HBV vaccine.

Results: At 4-5 years of age, $85.3 \%$ of subjects had persisting anti-HBs antibody concentrations $\geq 10 \mathrm{mlU} / \mathrm{mL}$, rising to $98.6 \%$ after the HBV challenge dose. All but 12 subjects (95.8\%) achieved post-challenge anti-HBs concentrations $\geq 100 \mathrm{mIU} / \mathrm{mL}$. The post-challenge anti-HBs GMC rose by 100 -fold compared to pre-challenge concentrations. An anamnestic response to the HBV vaccine challenge was observed in $96.8 \%$ of subjects, including 17/21 (81.0\%) of children with initially undetectable antibodies $(<3.3 \mathrm{mlU} / \mathrm{mL})$. All but 4 of 42 subjects $(90.5 \%)$ with anti-HBs antibodies $<10 \mathrm{mlU} / \mathrm{mL}$ prior to the challenge dose, achieved seroprotective levels afterwards. A 4-fold rise in antibody concentration after the challenge dose was observed in 259/264 (98.1\%) of initially seropositive subjects. The magnitude of the post-challenge responses was proportional to pre-challenge anti-HBs levels. No serious adverse events were reported during the study.

Conclusion: The combined DTPa-HBV-IPV/Hib vaccine induced lasting immune memory against hepatitis B. Long term protection afforded by DTPa-HBV-IPV/Hib is likely to be similar to that observed following priming with monovalent HBV vaccines.

Trial registration: http://www.clinicaltrials.gov 106789 NCT00411697

\section{Background}

Achieving high routine vaccination coverage against hepatitis B in infancy is considered the highest priority for hepatitis B prevention by the World Health Organization (WHO) [1]. Universal Infant vaccination as the primary prevention strategy was adopted by the WHO in 1988 [2], after the failure of vaccination strategies targeting only at-risk groups [3,4]. Infant vaccination has the greatest impact on preventing chronic hepatitis B

\footnotetext{
* Correspondence: volker.schuster@medizin.uni-leipzig.de

${ }^{5}$ University Hospital for Children and Adolescents, Leipzig, Germany
}

and its subsequent complications [1]. Furthermore, maintaining high vaccine coverage is more sustainable in infants than in adolescents who are difficult to reach and frequently poorly compliant [3,5-7].

Combination vaccines for use in infancy have an increasingly important role in contributing to high levels of parental acceptance of vaccination. Combination vaccines reduce the number of injections required for full vaccination and improve the timeliness of vaccination, thereby contributing to maintaining high levels of vaccine coverage $[8,9]$. Several combined vaccines 
containing hepatitis B vaccine are currently commercially available, the largest of which is the hexavalent diphtheria-tetanus-pertussis-hepatitis B-inactivated poliomyelitis and Haemophilus influenzae type b conjugate vaccine (DTPa-HBV-IPV/Hib) manufactured by GlaxoSmithKline Biologicals (GSK, Rixensart, Belgium).

DTPa-HBV-IPV/Hib is licensed for primary vaccination of infants and for second year of life booster vaccination in many countries throughout the world, including all European Union countries. Previous clinical studies have shown DTPa-HBV-IPV/Hib to be well tolerated and immunogenic [10]. In particular, three dose primary vaccination with DTPa-HBV-IPV/Hib induces seroprotective antibody levels (anti-HBs $\geq 10 \mathrm{mIU} / \mathrm{mL}$ ) against hepatitis B in over $95 \%$ of subjects [10], comparable to results following monovalent hepatitis $B$ vaccines $[10,11]$. This study expands upon these previous reports of DTPa-HBV-IPV/Hib by assessing the persistence of immunological memory in children between 4 and 5 years of age who had been previously primed and boosted with four doses of DTPa-HBV-IPV/Hib in their first two years of life.

\section{Methods}

The study was an open-label serological follow up study (http://www.clinicaltrials.gov 106789 NCT00411697) conducted in 27 centers in Germany, between 19 December 2006 and 14 May 2007. The study was conducted according to Good Clinical Practice guidelines, the Declaration of Helsinki, and applicable German laws. The study protocol was approved by Ethik-Kommission der Landesärztekammer Baden-Württemberg, Jahnstraße 40, 70597 Stuttgart. Written informed consent was obtained from parents/guardians before enrolment.

All subjects were healthy and previously vaccinated with four doses of DTPa-HBV-IPV/Hib (Infanrix hexa $a^{\text {ma }}$; GSK Biologicals) administered via routine immunization procedures in Germany. The recommended infant vaccination schedule in Germany is at 2, 3 and 4 months of age. Since strict adherence to the schedule could not be guaranteed, subjects were to have received three primary vaccination doses by 9 months of age and one booster dose received between 11 and 18 months of age. Subjects who had received hepatitis $B$ vaccination at birth, or any previous hepatitis $B$ booster vaccination after administration of the fourth DTPa-HBV-IPV/Hib dose in the second year of life were excluded.

Enrolled children received a single challenge dose of monovalent pediatric hepatitis B vaccine $\left(\right.$ Engerix $^{\mathrm{Tm}}-\mathrm{B}$ Kinder, containing $10 \mu \mathrm{g}$ recombinant hepatitis B surface antigen [HBs], GSK) administered intramuscularly into the deltoid. A blood sample was collected before and one month after the hepatitis B challenge dose.
Anti-HBs antibodies were measured using GSK Biologicals' in-house ELISA with a cut-off of $3.3 \mathrm{mIU} / \mathrm{mL}$ defining seropositivity [12]. Anti-HBs antibody concentrations $\geq 10 \mathrm{mIU} / \mathrm{mL}$ were considered to be seroprotective.

Serious adverse events occurring within 30 days of vaccination were recorded.

The primary analysis of immunogenicity was conducted on the according-to-protocol (ATP) immunogenicity cohort comprising subjects who complied with protocol defined procedures and criteria, who received the challenge hepatitis $B$ vaccine dose, and for whom immunogenicity data were available.

The percentage of subjects with anti-HBs antibody concentrations $\geq 10 \mathrm{mIU} / \mathrm{mL}$ and $\geq 100 \mathrm{mIU} / \mathrm{mL}$, with exact $95 \%$ confidence intervals $(\mathrm{CI})$, prior to and one month after the challenge dose, was calculated. Persistence of immunological memory shown by the ability to mount an anamnestic response to a challenge dose of hepatitis $B$ vaccine, was defined as anti-HBs concentrations $\geq 10 \mathrm{mIU} / \mathrm{mL}$ in subjects seronegative (i.e. antiHBs concentrations $<3.3 \mathrm{mIU} / \mathrm{mL}$ ) before the challenge and as an increase of at least 4-fold in anti-HBs concentrations in subjects seropositive (anti-HBs concentrations $\geq 3.3 \mathrm{mIU} / \mathrm{mL}$ ) before the challenge.

\section{Results}

In total, 301 subjects were enrolled and vaccinated. The ATP immunogenicity cohort consisted of 286 subjects. Subjects were eliminated from the ATP immunogenicity analysis because they had received forbidden vaccines; had not received the full DTPa-HBV-IPV/Hib course in infancy; had not complied with the blood sampling schedule; had missing serological data; had blood samples that could not be reliably identified; or because they had tested positive to anti-hepatitis B core antigen (one subject, $0.3 \%$ ).

The mean age of subjects in the ATP immunogenicity cohort was 4.5 years (standard deviation [SD] 0.5), of which $51.7 \%$ (148) were female. The majority of subjects were Caucasian.

Prior to the booster dose, $85.3 \%$ (250/293) of DTPaHBV-IPV/Hib primed and boosted subjects had persisting anti-HBs levels $\geq 10 \mathrm{mIU} / \mathrm{mL}$. This rose to $98.6 \%$ $(282 / 286)$ after the HBV challenge dose (Table 1) and the post-challenge anti-HBs antibody GMC rose by $100-$ fold compared to pre-challenge concentrations. All but 12 subjects $(95.8 \%)$ achieved post-challenge anti-HBs antibody concentrations $\geq 100 \mathrm{mIU} / \mathrm{mL}$.

An anamnestic response to the hepatitis $B$ vaccine challenge was observed in $96.8 \%$ [95\% CI 94.1; 98.5] of subjects $(276 / 285)$. Of 21 subjects whose pre-challenge anti-HBs antibody level was below $3.3 \mathrm{mIU} / \mathrm{mL}$ (i.e. seronegative), 17 (81.0\%) reached the $10 \mathrm{mIU} / \mathrm{mL}$ 
Table 1 Anti-HBs antibody responses before and one month after challenge dose (ATP Cohort for immunogenicity)

\begin{tabular}{|c|c|c|c|c|c|c|c|c|c|c|c|c|}
\hline \multirow[b]{3}{*}{ Time point } & \multirow[b]{3}{*}{$\mathrm{N}$} & \multicolumn{9}{|c|}{ Seropositivity/Seroprotection rates } & \multirow{2}{*}{\multicolumn{2}{|c|}{ GMC }} \\
\hline & & \multicolumn{3}{|c|}{$\geq 3.3 \mathrm{mlU} / \mathrm{mL}$} & \multicolumn{3}{|c|}{$\geq 10 \mathrm{mlU} / \mathrm{mL}$} & \multicolumn{3}{|c|}{$\geq 100 \mathrm{mlU} / \mathrm{mL}$} & & \\
\hline & & $\mathrm{n}$ & $\%$ & {$[95 \% \mathrm{Cl}]$} & $n$ & $\%$ & {$[95 \% \mathrm{Cl}]$} & $\mathrm{n}$ & $\%$ & {$[95 \% \mathrm{Cl}]$} & Value & {$[95 \% \mathrm{Cl}]$} \\
\hline Pre & 285 & 264 & 92.6 & {$[89.0 ; 95.4]$} & 243 & 85.3 & {$[80.6 ; 89.2]$} & 134 & 47.0 & {$[41.1 ; 53.0]$} & 83.7 & {$[66.8 ; 104.9]$} \\
\hline Post & 286 & 284 & 99.3 & [97.5: 99.9$]$ & 282 & 98.6 & [96.5: 99.6$]$ & 274 & 95.8 & [92.8: 97.8$]$ & 8656.1 & [6560.7: 11420.9$]$ \\
\hline
\end{tabular}

GMC = geometric mean antibody concentration. $\mathrm{N}=$ number of subjects with available results. $\mathrm{n} / \%=$ number/percentage of subjects with antibody concentrations above the specified cut-off. $95 \% \mathrm{Cl}=$ exact $95 \%$ confidence interval. Pre = prior to the administration of challenge dose, Post $=$ one month after the challenge dose

seroprotection cut off after the challenge dose. Of 42 subjects who had anti-HBs antibody concentrations below $10 \mathrm{mIU} / \mathrm{mL}$ prior to the challenge dose, all but 4 (90.5\%) achieved seroprotective anti-HBs levels after the challenge (Table 2). Of 264 subjects with detectable anti-HBs antibodies prior to the challenge dose, 98.1\% (259) had at least a 4-fold increase in antibody concentrations following the challenge dose. The magnitude of the post-challenge responses was proportional to prechallenge anti-HBs levels (Table 2, Figure 1).

No serious adverse events were reported during the study.

\section{Discussion}

Primary and booster vaccination in the first two years of life with DTPa-HBV-IPV/Hib induced long lived immunological memory against hepatitis $B$ in subjects between 4 and 5 years of age who had been primed and boosted via routine pediatric services in Germany. These data are remarkably similar to data obtained from cohorts of subjects 4 to 6 years of age primed with DTPa-HBV-IPV/Hib in clinical trial settings in Germany, where adherence to the primary and booster vaccination schedule was likely to have been more strictly enforced [13,14]. In these studies, anti-HBs antibody levels associated with seroprotection were observed between $86.0 \%$ and $91 \%$ of DTPa-HBV-IPV/Hib trialvaccinated subjects at 4-6 years of age [13,14], and an anamnestic response following HBV vaccine challenge was observed in $95.7 \%$ [14]. A recent follow-up study conducted in Italy in children in the third year of life, primed with DTPa-HBV-IPV/Hib at 2, 3 and 11/12 months of age, also showed high persisting seroprotective anti-HBs levels (96.0\% of subjects) and anamnestic

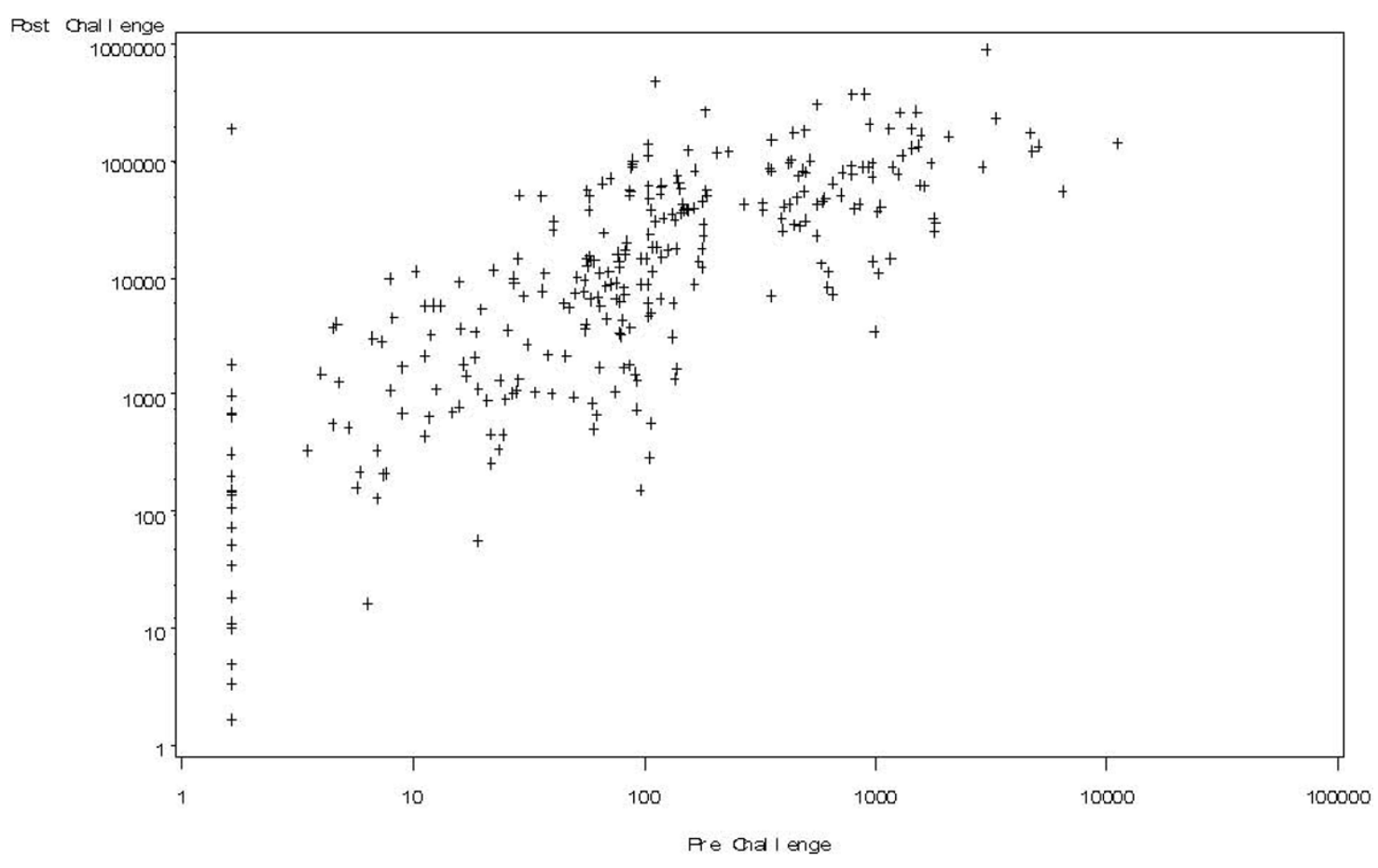

Ant $i-H E$ s anti body concentrati ons (MUNM)

Figure 1 Relationship between anti-HBs antibody concentrations before and after HBV challenge (ATP Immunogenicity cohort). 
Table 2 Anti-HBs antibody responses one month after HBV challenge by pre-vaccination status (ATP Cohort for immunogenicity)

\begin{tabular}{|c|c|c|c|c|c|c|c|c|c|c|c|c|}
\hline \multirow[b]{3}{*}{ Pre-challenge status } & \multirow[b]{3}{*}{$\mathrm{N}$} & \multicolumn{6}{|c|}{ Post challenge } & \multirow{2}{*}{\multicolumn{3}{|c|}{$\geq 100 \mathrm{mlU} / \mathrm{ml}$}} & \multirow{2}{*}{\multicolumn{2}{|c|}{ GMC }} \\
\hline & & \multicolumn{3}{|c|}{$\geq 3.3 \mathrm{mlU} / \mathrm{ml}$} & \multicolumn{3}{|c|}{$\geq 10 \mathrm{mlU} / \mathrm{ml}$} & & & & & \\
\hline & & $\mathrm{n}$ & $\%$ & $95 \% \mathrm{Cl}$ & $\mathrm{n}$ & $\%$ & $95 \% \mathrm{Cl}$ & $\mathrm{n}$ & $\%$ & $95 \% \mathrm{Cl}$ & value & $95 \% \mathrm{Cl}$ \\
\hline$<3.3 \mathrm{mlU} / \mathrm{ml}$ & 21 & 19 & 90.5 & {$[69.6 ; 98.8]$} & 17 & 81.0 & {$[58.1 ; 94.6]$} & 11 & 52.4 & {$[29.8 ; 74.3]$} & 85.4 & {$[24.7 ; 295.0]$} \\
\hline$\geq 3.3 \mathrm{mlU} / \mathrm{ml}$ & 264 & 264 & 100 & {$[98.6 ; 100]$} & 264 & 100 & {$[98.6 ; 100]$} & 262 & 99.2 & {$[97.3 ; 99.9]$} & 12524.1 & [9907.2; 15832.2] \\
\hline$<10 \mathrm{mlU} / \mathrm{ml}$ & 42 & 40 & 95.2 & {$[83.8 ; 99.4]$} & 38 & 90.5 & {$[77.4 ; 97.3]$} & 31 & 73.8 & {$[58.0 ; 86.1]$} & 249.8 & {$[116.9 ; 534.1]$} \\
\hline$\geq 10 \mathrm{mlU} / \mathrm{ml}$ & 243 & 243 & 100 & {$[98.5 ; 100]$} & 243 & 100 & {$[98.5 ; 100]$} & 242 & 99.6 & {$[97.7 ; 100]$} & 16010.1 & [12812.2; 20006.2] \\
\hline$<100 \mathrm{mlU} / \mathrm{ml}$ & 151 & 149 & 98.7 & {$[95.3 ; 99.8]$} & 147 & 97.4 & {$[93.4 ; 99.3]$} & 139 & 92.1 & {$[86.5 ; 95.8]$} & 2119.3 & {$[1475.8 ; 3043.4]$} \\
\hline$\geq 100 \mathrm{mlU} / \mathrm{ml}$ & 134 & 134 & 100 & {$[97.3 ; 100]$} & 134 & 100 & {$[97.3 ; 100]$} & 134 & 100 & {$[97.3 ; 100]$} & 42433.2 & [34084.1; 52827.5] \\
\hline
\end{tabular}

$\mathrm{N}=$ number of subjects with available results. $\mathrm{n} / \%=$ number/percentage of subjects with antibody concentrations above the specified cut-off. $95 \% \mathrm{Cl}=$ exact $95 \%$ confidence interval.

responses to a challenge $\mathrm{HBV}$ vaccine dose in those with concentrations $<10 \mathrm{mIU} / \mathrm{mL}$ [15]. Together, these results provide strong evidence for the immunogenicity of the hepatitis B component of DTPa-HBV-IPV/Hib in routine use.

Booster doses of hepatitis B vaccine after primary vaccination in immunocompetent individuals are currently not recommended by either the WHO [16] or the European Consensus group on Hepatitis B Immunity [17]. Decrease of antibody concentrations below seroprotection level or even below detection levels is not considered as an indicator of loss of protection. Persisting protection against clinical hepatitis $B$ disease and chronic hepatitis B carriage has been shown to last for up to two decades [17-20].

A potential limitation of the study is that the analysis was performed, as pre-planned in the protocol, on ATP immunogenicity cohort. However, fewer than $5 \%$ of subjects (15/301) were eliminated from the ATP cohort, and of these, blood samples were not available or could not be reliably identified for 5 subjects. It is therefore unlikely that the conclusions from an analysis of the Total cohort, with data from only 10 additional subjects, would differ from the ATP analysis. The findings of the present study are important because they confirm the medium term immunogenicity of hepatitis $B$ vaccination by using a hexavalent combination vaccine in subjects from a low endemicity region who are unlikely to have been exposed to hepatitis B, and who were primed with a large combination vaccine. Further serological followup studies of older individuals until age 15 years, who received four consecutive doses of DTPa-HBV-IPV/Hib as primary and booster vaccination in routine vaccination practice are planned, and will be performed as vaccinated cohorts become older.

\section{Conclusions}

In conclusion, primary and booster vaccination with DTPa-HBV-IPV/Hib in infancy induces sustained seroprotection against hepatitis B up to the age of 4-6 years. A strong response to hepatitis $B$ vaccine challenge at this age was demonstrated, indicative of persisting immune memory, even in subjects who had lost circulating antibodies. Longer follow-up is required to ascertain the presence of immune memory at later ages.

\section{Acknowledgements}

The authors thank Joanne Wolter and Julia Donnelly for editorial assistance in preparing this manuscript and Mohammed Benaata and Valérie Haezebroeck for laboratory assays.

Infanrix hexa and Engerix-B are trademarks of the GlaxoSmithKline group of companies.

\section{Author details}

${ }^{1}$ Medical Practice, Werderstr. 3, 88348 Bad Saulgau, Germany. ${ }^{2}$ Clinical Statistics, GlaxoSmithKline Biologicals, Bangalore, India. ${ }^{3} \mathrm{Med}$. Fachbereich Impfstoffe, GlaxoSmithKline Biologicals, Munich, Germany. ${ }^{4}$ Clinical Department, GlaxoSmithKline Biologicals, Rixensart, Belgium. ${ }^{5}$ University Hospital for Children and Adolescents, Leipzig, Germany.

\section{Authors' contributions}

GR participated in the design of the study and performed the statistical analysis. MS, VS, BG, OVM, J-MJ conceived of the study, and participated in its design and coordination and helped to draft the manuscript. All authors read and approved the final manuscript.

\section{Competing interests}

This study was funded by GlaxoSmithKline Biologicals. Drs B Gartner, R Gunasekaran, JM Jacquet and O Van Der Meeren are employees of GlaxoSmithKline Biologicals. VS received investigator fees from GSK. All authors participated in the design or implementation, analysis and interpretation of the study, the writing of the report and the decision to submit the manuscript for publication.

Received: 24 July 2009

Accepted: 15 January 2010 Published: 15 January 2010

\section{References}

1. World Health Organization: Hepatitis B vaccines WHO position paper. WER 2004, 79:255-263.

2. World Health Organization: Progress in the control of viral hepatitis: memorandum from a WHO meeting. Bull WHO 1988, 66:443-455.

3. Lawrence $M H$, Goldstein MA: Hepatitis B immunization in adolescents. $J$ Adolesc Health 1995, 17:234-243.

4. Centers for Disease Control and Prevention: Hepatitis B Virus: A comprehensive strategy for eliminating transmission in the United States through universal childhood vaccination: Recommendations of 
the Immunization Practices Advisory Committee (ACIP). MMWR 1991, 40(RR-13):1-19.

5. Brabin L, Greenberg DP, Hessel L, Hyer R, Ivanoff B, Van Damme P: Current issues in adolescent immunization. Vaccine 2008, 26:4120-34.

6. Heininger U, Loos K, Lorenz I, Rascher W: Compliance with recommended immunizations in adolescents. Eur J Pediatr 2006, 165:671-676.

7. Vandermeulen C, Roelants M, Theeten H, Depoorter AM, Van Damme P, Hoppenbrouwers K: Vaccination coverage in 14-year-old adolescents: documentation, timeliness, and sociodemographic determinants. Pediatrics 2008, 121:e428-434.

8. Freed GL, Cowan AE, Clark SJ, Santoli J, Bradley J: Use of a new combined vaccine in pediatric practices. Pediatrics 2006, 118:e251-257.

9. Kalies H, Grote V, Verstraeten T, Hessel L, Schmitt HJ, von Kries R: The use of combination vaccines has improved timeliness of vaccination in children. Pediatr Infect Dis J 2006, 25:507-512.

10. Zepp F, Schmitt J, Cleerbout J, Verstraeten T, Schuerman L, Jacquet J-M: Review of eight years of experience with Infanrix hexa (DTPa-HBV-IPV/ Hib hexavalent vaccine). Exp Rev Vaccines 2009, 8:663-679.

11. Lim FS, Han HH, Jacquet JM, Bock HL: Primary vaccination of infants against hepatitis $B$ can be completed using a combined hexavalent diphtheria-tetanus-acellular pertussis-hepatitis B-inactivated poliomyelitis-Haemophilus influenzae type B vaccine. Ann Acad Med Singapore 2007, 36:801-806.

12. Cambron P, Jacquet J-M, Hoet B, Lievens M: Development and Technical and Clinical Validation of a Quantitative Enzyme-Linked Immunosorbent Assay for the Detection of Human Antibodies to Hepatitis B Surface Antigen in Recipients of Recombinant Hepatitis B Virus Vaccine. Clin Vacc Immunol 2009, 16:1236-1246.

13. Heininger U, DTP-HBV-IPV-059 Study Group, DTP-HBV-IPV-096 Study Group: Booster immunization with a hexavalent diphtheria, tetanus, acellular pertussis, hepatitis B, inactivated poliovirus vaccine and Haemophilus influenzae type $b$ conjugate combination vaccine in the second year of life: safety, immunogenicity and persistence of antibody responses. Vaccine 2007, 25:1055-1063.

14. Zinke M, Kappes R, Kindler K, Paulus-Koschik A, Goering U, Disselhoff J, Soemantri P, Grunert D, Laakmann H, Gunasekaran R, Gartner B, Jacquet JM: Immune memory to hepatitis $B$ virus in 4-to-9-year old children vaccinated in infancy with four doses of hexavalent DTPa-HBV-IPV/Hib vaccine. Human Vaccines 2009, 5:592-598.

15. Giambi C, Bella A, Barale A, Montu D, Marchisio M, Oddone M, Zito S, Rapicetta M, Chionne P, Madonna E, degli Atti ML: A cohort study to evaluate persistence of hepatitis B immunogenicity after administration of hexavalent vaccines. BMC Infect Dis 2008, 8:100.

16. World Health Organization: Core information for the development of immunization policy. Document WHON\&B/02.28, 2002 Geneva, WHO 2002.

17. European Consensus Group on Hepatitis B Immunity: Are booster immunisations needed for lifelong hepatitis B immunity?. Lancet 2000, 355:561-565

18. Alfaleh F, Alshehri S, Alansari S, Aljeffri M, Almazrou Y, Shaffi A, Abdo AA: Long-term protection of hepatitis B vaccine 18 years after vaccination. $J$ Infect 2008, 57:404-409.

19. Sande van der MA, Waight PA, Mendy M, Zaman S, Kaye S, Sam O, Kahn A, Jeffries D, Akum AA, Hall AJ, Bah E, McConkey SJ, Hainaut P, Whittle HC: Long-term protection against HBV chronic carriage of Gambian adolescents vaccinated in infancy and immune response in HBV booster trial in adolescence. PLOS ONE 2007, 2:e753.

20. Van Damme $P$, Van Herck K: A review of the long-term protection after hepatitis A and B vaccination. Travel Med Infect Dis 2007, 5:79-84.

\section{Pre-publication history}

The pre-publication history for this paper can be accessed here:http://www. biomedcentral.com/1471-2334/10/9/prepub

\section{doi:10.1186/1471-2334-10-9}

Cite this article as: Steiner et al:: Lasting immune memory against hepatitis B in children after primary immunization with 4 doses of DTPa-HBV-IPV/Hib in the first and $2^{\text {nd }}$ year of life. BMC Infectious Diseases 2010 10:9. 